\title{
Implementation of Agile Methodology in Developing Countries: Case Study in Lebanon
}

\author{
Scarlet Rahy Rahy ${ }^{1 *[0000-1111-2222-3333]}$, and Julian M. Bass ${ }^{10000-0002-0570-7086]}$ \\ ${ }^{1}$ University of Salford, Greater Manchester, UK S.Rahy@edu.salford.ac.uk; J.Bass@sal- \\ ford.ac.uk http://www.salford.ac.uk
}

\begin{abstract}
Researchers have become interested in agile approaches to information systems development, because of their potential to improve product quality and increase productivity. While often associated with project orchestration in software development, agile methods have a wider potential application within ICT4D.

Our interest, in this research, is to explore how practitioners in developing countries describe factors that either enable or impede the adoption of agile information system development methods. We chose Lebanon to investigate this question, because it is a center for outsourcing software development in Middle Eastern countries. We employed a qualitative research approach by conducting 31 semi-structured recorded interviews with practitioners in three Lebanese software development companies. We employ an analytical model which interlinks institutional theory, the capability approach, and ICTs to explore creating software using agile methodology.

The analysis revealed positive reinforcement between three factors that improve the effectiveness of agile methods including the inclusive and detailed sprint planning, rich variety of stakeholder communication tactics, and teams that embrace a rich variety of communication technologies that overcome challenges presented by geographical distance. On the other hand, we also discovered impediments to agile adoption including team and management misunderstandings of agile methodology and the current political and economic crisis in the country.

The model revealed the low investment of companies in ICT. The lack of knowledge of agile ceremonies roles and artefacts created gaps in the implementation process. Also, the model highlighted bottlenecks that should be crucially tackled such as the lack of customer involvement and risk mitigation towards external factors.
\end{abstract}

Keywords: Agile Methodology, ICT4D, Institution Theory, Capability Approach, Developing Countries, Lebanon.

\section{Introduction}

The agile methods are based on values and philosophies developed in the Agile Manifesto [9], which promotes continuous customer involvement and encourages feedback. Agile information systems development is associated with the potential improvement of team productivity and software product quality [2]. Agile is increasingly expanding its influence from software development project planning to applications within the information and communication technology for development (ICT4D). Thus, researchers have become interested in the study of this field.

As in the global market, the demand for software development in developing countries is rising. Software development providers aim to enhance the quality and productivity of their development process. This study chooses Lebanon as a research site since 
Lebanon falls in UNDP's list of development aid recipient countries and is a source for outsourcing software in the Middle East and Gulf region [13, 15].

This paper describes and analyzes Agile Software Development in the developing country's software development industry using a model originally presented in [7]. The model created by [7] introduces an analytical framework for information and communication technology drawing on the capabilities approach and institutional theory. This combination uses institutional theory, focusing on roles, norms and organizational structures, and the capabilities approach, focusing on the achievement of goals and desires to account for the technology driven change in institutions in developing countries. It highlights the inhibitors and exciters between the model's different elements to create an analytical framework for ICT4D.

Three dimensions connect each of the elements that constitute the analytical platform. We explore how the human capabilities and their understanding of agile affect the roles and organization's structure in relation to agile implementation of ceremonies and artefacts in ICT context. This paper fills the gap and answers the below research question: What do practitioners in developing countries describe as factors that either enable or impede the adoption of agile?

The study employs a qualitative research approach by conducting $31 \mathrm{semi}$-structured interviews with practitioners in three Lebanese software development companies. We employ an analytical model which interlinks institutional theory, the capability approach, and ICTs to explore creating software using agile methodology. The model aids in identifying the inhibiters and exciters in the process.

This paper is structured as follows: first we present a literature review on agile software development and the research model. Then, we illustrate the methodology used to gather and analyze data. Then, we present an implementation of the model in the studied context followed by a discussion. Finally, conclusions and future work are presented.

\section{Agile software development in developing countries}

The agile methodology promotes continuous involvement of the customer in the development process [14]. In addition to software engineering, agile development has attracted attention in information systems and project management disciplines [11]. Agile methods are associated with increased level of productivity [12]. Conventionally, agile methods are comprised of ceremonies, such as stand-up meetings, retrospectives and customer demos, artefacts, which fall under five categories: feature, sprint, release, product and development programmed governance [5] and roles, which include selforganizing teams, product owners, and scrum masters. Agile practices may be tailored by practitioners to fit their context, large scale, regulated or disciplined environment[6].

\subsection{Agile Implementation in Developing Countries}

Developing countries have been implementing agile methods in their software development process. Practitioners are aware of the benefits of agile and stress on the importance of its implementation [18]. Gaps between the developers and the end-users 
hinder the development process $[3,10]$. This is due to the end-user's IT skill level and communication pattern with developers [3].

For instance, a study conducted in Egypt revealed the positive effects on the software development process and customer satisfaction when adopting agile methodology [16]. Simultaneously, challenges were detected such as, high pressure on developers, inadequate use of effort estimation, and lack of sprint planning. Also, a study of an in-house software development for a Lebanese university revealed the lack of support from the educational sector [21, 22] and poor ICT structure [21]. The country's poor ICT infrastructure and lack of government plan for the IT industry stand as barriers to growth [24]. There is scarcity in the literature studying the benefits of implementing agile, its challenges, practices, and information flows in the Lebanese context. Thus, there is a large scope for conducting new studies to understand agile in developing countries, through studying the Lebanese context. This research aims at providing an understanding of agile software development in developing countries in relation to the Technology Institutions and Capabilities model's elements.

Agile teams in developing countries have the potential to enhance their software development industry through effectively applying agile methods. The analysis of geographically separated teams in Kenya and The Netherlands showed how teams may use their differences in order to benefit and learn from each other's experiences [17]. Team members at The Netherlands learned and enhanced their agile process by observing agile being implemented in Kenya.

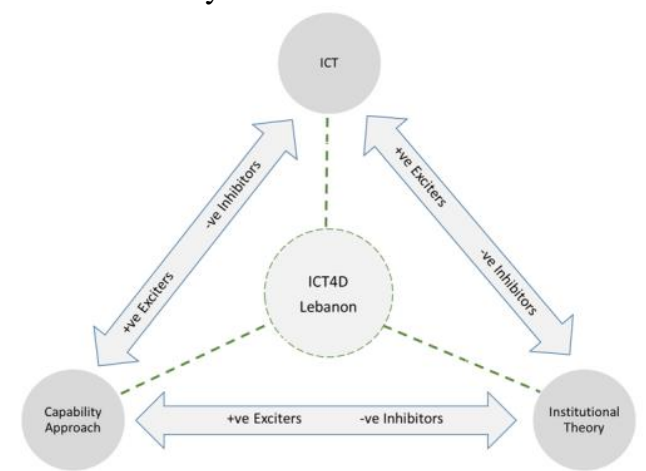

Fig. 1: Technology Institutions and Capabilities model adapted from Bass et al. (2013)

\subsection{Research Model}

This research is based on the conceptual model, Technology Institutions and Capabilities model (TIC) created by [7] is shown in Fig.1. The framework identifies a relationship between institutional theory, the capability approach, and ICT. In the context of the model, ICT is the technology used to 'deliver human centered development', the capability approach looks into the freedom given to individuals to expand their abilities using ICT, and institutional theory focuses on structure, technology, and institutional driven change. The bidirectional arrows between the model's elements represent the 
influences and interrelationships. The positive ones are labeled as exciters and the negatives ones are labeled as inhibitors. The model is used here to identify the strength and weakness of agile software development implementation in developing countries.

\section{Methodology}

The foundation of this research is based on the model presented by [7]. This model enables us to present a framework that combines institutional theory and the capability approach in relation to ICT and analyze the influences along the three different elements. This research used a case study approach, guided by [19], to explore the practitioner's perception on agile implementation in the Lebanese software development industry. The case study replicates the investigation in three different software development companies. Interviews were conducted with 31 respondents who were ensured confidentiality and anonymity. The following section introduces the research sites, data collection and data analysis process.

\subsection{Research sites}

The agile software development industry is spreading across developing countries. Lebanon is considered as a case study for this research since it's a developing country and it outsources software to Middle Eastern and gulf countries [13, 15]. Thus, we chose practitioners involved in agile software development form 3 different research sites. The three sites were labelled LEB1, LEB2, and LEB3 to protect their anonymity.

LEB1 is a Lebanese based software development company providing solutions in areas of banking, analytics, technology, academy, insurance, retail, healthcare, and multimedia. LEB1 has its main headquarters in Mount Lebanon, and two other branches in North Lebanon, and Bekaa Valley. LEB2 is a software development company that provides technological solutions for clients in Lebanon and abroad. LEB2 develops on-demand and customized software using agile. LEB3 is a Lebanese based software development company that develops apps and websites for its clients.

\subsection{Data collection}

The primary source of data collection is face-to-face interviews conducted in person. A list showing the participants' research sites, roles, and respective location is found in [8]. The participants' selection was done with the help of company representatives. The interviews ranged from 30 minutes to 1 hour and 10 minutes with 45 minutes being typical. The data collected was obtained from semi-structured open-ended interviews. Probing questions were used to encourage participants to discuss any new topics that were not included in the interview guide. The interview guide and consent form used for interviews may be viewed at [4]. All interviews were recorded after obtaining the practitioners' consent. Field notes were taken during the interviews by the researcher. Then, interviews were transcribed manually since it ensures correct transcription and 
reminds the interviewer of the social and emotional aspects that occurred during the interview [23] thus effectively conducting the data collection stage [1].

\subsection{Data analysis}

For the transcribed interviews, we performed open coding, applied constant comparison methods, identified core categories, and memoed the data. All transcribed data was imported to an analyzing tool Nvivo 11. Open coding includes the analysis of data and exclusion of prior judgment to produce the maximum number of concepts. Line-by-line open coding approach was used on the transcribed interviews. Codes from each interview were compared from codes arising from the same and other interviews. This constant comparison technique enabled the grouping of codes that constitute concepts. Then, each key point is assigned a code titled with a phrase that summarizes it. From these, interview concepts arise and are grouped into categories which then form the main categories. We then chose the topics that align with the model of our choice. We chose the core categories and perform selective coding according to the framework of the model explained in the previous section. Our analysis involves mapping of our case onto the conceptual model supported by textual description.

\section{$4 \quad$ Findings}

We now explore how the TIC model can be used to understand agile implementation in Lebanon. This will enable us to explore how each of the model's elements when strengthened can decrease the inhibitors and increase the exciters in each case.

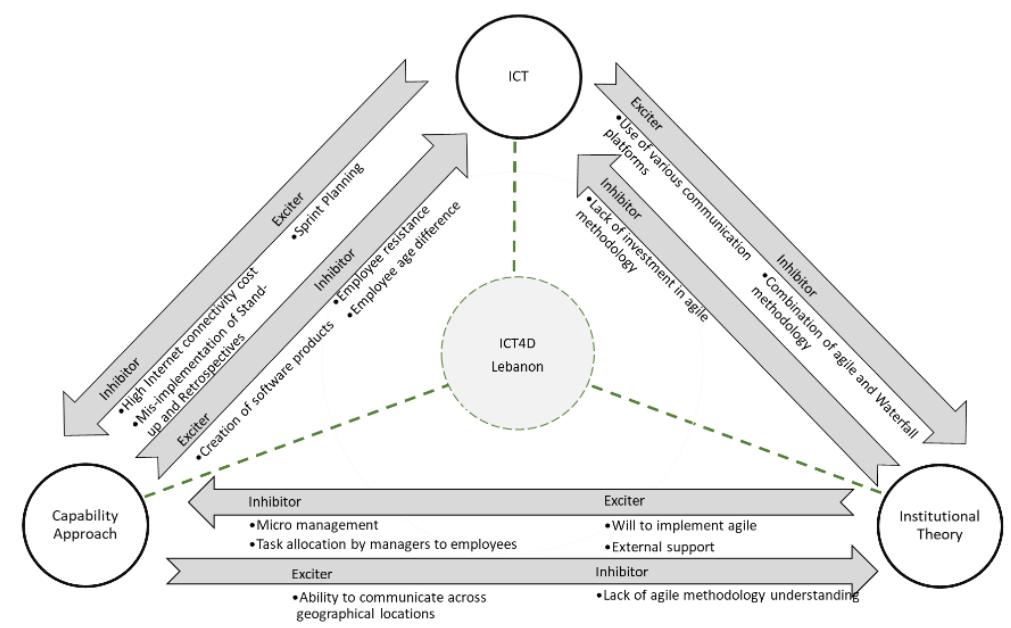

Fig.2. TIC model adapted to the Lebanese context

In our scenario, the capabilities approach is the people's ability to fully understand and correctly implement agile. ICT is software development methods used along with the agile ceremonies implemented and artefacts created. Institutional theory includes 
the roles of the employees and the structure of the organization. Fig. 2 reveals the model adapted to the Lebanese context. Each of the three dimensions is explained thoroughly and the respective inhibiters and exciters are shown.

\subsection{Dimension A: Capabilities to Institutions}

A major obstacle towards applying agile is understanding what agile methodology is. Using the TIC analytical framework, we find this as an inhibitor from capabilities to building the software development institution. Being a new concept in the Lebanese software development industry, understanding agile is a major struggle for employees. First of all, implementing agile was suggested by either employees who discovered the agile concept while researching or the CEO who heard about the concept in a different company having no prior experience or knowledge. S6 explained: "While I was researching for a project, I found out about agile. I presented this concept to the team leader and he liked the idea. So the company decided to turn into agile." Understanding agile and its importance was especially difficult for non-developers. A2 explained: "Business development and marketing team didn't understand agile's importance".

On the other hand, the ability to communicate across diverse geographical location acts as an exciter from capabilities to institutions. Interviews revealed that teams were located in different geographical locations while working on the same project. These team members come from different culture, tradition, and religion, which highly affects the person's character in Lebanon. Communication between these members is smooth. Surprisingly, from all the interviewed practitioners, who worked with outsourced employees or with team members in different company branches, none claimed that they are currently facing communication difficulties. Team members would join for meetings virtually through different communication platforms. A1 explained: "Every morning at 9, everyone had to participate in the stand up and the employees outside the office would join in virtually." Different communication platforms were used to communicate with employees who are outside the office such as slack, email, calls, video conferencing. A6 said: "Every time we needed something from someone we used slack, whether they are in the office or outside. The communication was very easy." Sometimes, urgent matter arise and employees from different geographical locations will have to join at the headquarters. Managers try to minimize the movement between branches to decrease time loss time; since the branches are located in different districts, no public transportation is available in Lebanon, and traffic jams are major.

\subsection{Dimension A: Institutions to Capabilities}

In the opposing direction, micro management by the CEO and allocation of tasks by the managers are inhibitors from institutions to capabilities. First, dealing with the pressures of the upper management is one of the main challenges faced by employees. A2 said: "Honestly, the biggest challenge faced is the upper management." Practitioners revealed how the $\mathrm{CEO}$ was micro-managing and continuously involved in the process.

In regards to communication, the CEO requested to know all the details of every project. So the CEO would request a morning meeting to be informed of all the details. 
V9 expressed their opinion on these meetings: "Instead of taking 20 to 30 minutes for each daily meeting, we would use it for our work to be done." These morning meeting will last longer than advised. Managers complained from the CEO's interference in how they lead their team and plan and execute their upcoming tasks. A4 described: "I am not given the space to implement the work independently. So I had the CEO assign tasks to my team without me knowing. He was deciding our next steps even if I didn't know." Thus, A4, team manager, and A7, team member, described how they would use WhatsApp and Slack in order to communicate without being interrupted by the CEO. They would plan their overall tasks and daily to-do lists.

Second, managers and team leaders assign tasks to the team members. V7 explained: "We assign the global tasks for each team and then each team leader will assign the tasks to his team." Occasionally, team leaders discuss the task distribution with their team members. This creates occasional tension between the team leaders and project managers. Also, when tasks are imposed, tension between team members rise and they prefer to choose the tasks they want to work on. As A7 describes: "I dislike the idea that our leaders allocate the tasks. I would like to choose the tasks I have to do." S6 added: "It would be motivating if I chose my own tasks from each sprint." When tasks are allocated, some team members object and some team leaders disregard it.

Conversely, the upper management are open to implement agile. This demonstrates positive intentions towards this method. Also, external support is provided through the Beirut Digital District (BDD) which is a project that aims at creating a hub for the digital community in Lebanon. Occasionally, BDD conducts workshops on agile software development. Few practitioners mentioned that they attended these workshops.

\subsection{Dimension B: Capabilities to ICT}

The employee age difference and resistance are inhibitors flowing from capabilities to ICT. First, the age difference between employees is a challenge for managers to overcome. An employee who has just joined the company may be more qualified than another employee in a senior position. V4 gives an example: "My challenge is how to say to a senior that a junior developer will be your supervisor." This difference in ICT knowledge creates tension between certain employees.

In addition, the employees' resistance has been a subject in the management world for decades. In our studied companies, resistance was detected from the employees as well as the upper management. Employee resistance was detected when implementing new methods or when assigning certain tasks. V1 claimed "Maybe they feel they are overwhelmed with the work load they have; they feel it is out of their roles and responsibilities; or they have some tension with other team members." Employee resistance was also detected when implementing agile methods especially amongst non-developers. A2 explained: "The business development team and the marketing team do not understand the importance of agile." Upper management resistance appeared when implementing new agile methods. A2 continued: "Honestly the upper management was my main struggle towards reaching agility."

On another hand, software products being created from the Lebanese companies to local and international markets are exciters from the capabilities to the ICT sector. The 
numerous success stories revealed by practitioners show how much the team's capabilities is enhancing the industry.

\subsection{Dimension B: ICT to Capabilities}

The inhibitors that flow from ICT to capabilities are the high internet connectivity costs and the improper implementation of agile ceremonies. In developing countries such as Lebanon the internet access is characterized by high costs and low quality. This sometimes hinders the process and increases the time for a certain task to be completed.

Through the interviews, it was apparent that practitioners either didn't implement standups or implemented them incorrectly. For instance, LEB1 didn't implement the standup meetings with the team members. Instead, morning meetings were held with the CEO, top managers and team leaders. V6 explained: "In this meeting, we put all our active items. The CEO starts and then asks each team leader what they have for the day." V8 indicated that the morning meeting takes around 30 minutes and: "They should have a different formulation. We discuss the project in general. It is sometimes repetitive." Another example is the stand-up held at LEB3 where all the members of the company, even the employees who weren't present in the company premises, would join in using Zoom. The time for each standup took from 20 to 30 minutes which was a "tiring way to start the day [الصبح من نهلك كنا]", according to A4. Similarly to LEB1, the CEO used to take the lead in the standup. After an all-inclusive meeting, the product owners and team leaders weren't able to conduct their own standup with their team members: "It was impossible for me to do that again with my team."

In addition, through our interviews, confusion was detected on retrospectives, even in the same company. When asked about retrospectives, 21 interviewees didn't know what retrospectives are. So, as the interviewer, I had to explain what happens during retrospectives and how they are conducted. S1 responded: "We did not do retrospectives; we didn't have formal evaluations. We just informally say what was wrong." As for the interviewees who responded to the question regarding retrospectives, they lacked a clear understanding of what they are. Every response was different from the other and misconception on retrospectives were clear even in the same company.

On the other hand, implementing sprint planning, an agile ceremony, acts as an exciter from the ICT to capabilities. Sprint planning was done by all interviewed practitioners. All practitioners identified its importance towards the success of the sprint.

\subsection{Dimension C: ICT to Institutions}

The lack of understanding of agile act as an inhibitor from ICT to institutions. This lack of knowledge led practitioners to implement a combination of methods, Waterfall and agile methods or tailored agile methods with traditional project management methodologies, and ineffective end-user involvement. As mentioned by V1: "Inside the agile iteration we use the Waterfall methodology by respecting the sequence of processes." The sequence is explained by collection of requirements, design, development, testing and delivery. Another example, the upper management needed to see a global picture 
of the requirements. The overall requirements were given in the traditional project management methods. V9 explained: "We get the requirements from the project manager." In addition, although the companies have no direct control over the end-user, the enduser's lack of involvement hinder the process. V15 expressed: "I've noticed with Lebanese clients is the staff's availability and commitment is not always as promised."

The various communication platforms used are seen as exciters from ICT to institutions. Communication is highly encouraged at the agile implementing software development companies. Diverse communication channels are open for employees at the company. The usage of communication channels depends on the needs and message delivered. If it is a simple update, then instant messaging platforms are used. If it is formal, then emails are sent and meetings are held. V4 explained: "Sometime, I have to send just an informal message; I use WhatsApp." A1 indicated that slack is one of the major communication platforms used. When practitioners need to communicate and are present in different geographical locations, they use online video conference call applications that are available free of charge.

\subsection{Dimension C: Institutions to ICT}

The lack of investment from institutions in agile software development acts as inhibitors towards building an agile environment. Of the three research sites, non had a certified agile coach who guides employees through agile implementation or a scrum master. A2 was responsible for implementing agile in LEB3 with no prior experience or certification. In LEB1, agile was tailored according to trial and error methods. V1 explained: "Unfortunately, we base our work on research that we do on our own. We never had a coach specialized in agile methodology. We do our own research and we let ourselves be guided at the same time by the real needs on the ground." The employees didn't thoroughly understand the concept of agile. For instance, some interviewee had no knowledge on what agile ceremonies and artefacts were. They knew general concepts such as individual interactions over processes and working software over comprehensive documentation.

\section{Discussion}

Since agile information systems development is associated with the potential improvement of team productivity and software product quality, we aim to explore how practitioners in developing countries describe factors that either enable or impede the adoption of agile information system development methods.

We use the TIC model to understand the exciters and inhibitors in each dimension in the agile software development industry in developing countries through our case study in Lebanon. We identified virtuous and vicious circles that enhance or diminish the role of agile methodology in the software development industry. The study identifies positive reinforcements between factors that improve the effectiveness of agile methods. An example of a virtuous circle is the exciter: inclusive and detailed sprint planning (ICT to capabilities) leading to the creation of software products (capabilities 
to ICT). The various communication tactics act as exciters when used along with a rich variety of communication technologies between teams across different geographical locations. Although our case isn't cross-country with no time difference, yet employees cross long distances in personal cars with no public transportation available. These exciters enable team members to overcome distance and religious and cultural differences.

Inhibitors create vicious circles that undermine the agile software development process. For example, lack of agile methodology understanding (capabilities to institutions), leads to task allocation by managers to employees (institutions to capabilities). In addition, we were able to identify loops that hinder the correct implementation of agile methodology. For instance, the combination of agile and waterfall methodology (inhibitor from ICT to institutions) leads to the task allocation by managers to employees (inhibitor from institutions to capabilities) which in turn lead to employee resistance (inhibitor from capabilities to ICT). These closed loops obstruct agile implementation.

We had a difficulty assigning a satisfying illustration of an exciter from institutions to ICT. In our case, this may be due to the current economic and political crisis in the country. Also, the recognition of the institution's role in support of ICT is less fortified.

Our findings are consistent with previous research conducted in Ethiopia, which show novice agile practitioners tend to mix plan-based and agile methods [18]. Also, companies experience gaps when implementing agile practices with missing roles, ceremonies and artefacts due to lack of staff, knowledge and finances. We also, found that, like in Egypt, novice agile practitioners find effort estimation challenging [16]. However, a surprising finding from our research was the enthusiastic adoption of communication technologies to overcome challenges of geographical distance

The software development industry in Lebanon needs to tackle in parallel the social and technical issues [10]. The relation between ICT and capabilities approach in the model stress the latter. This reveals the need to mitigate for the social bottlenecks that companies face. These include the lack of involvement of the end-user in the development process $[3,20]$ and the political and economic issues the developing countries face, especially Lebanon in the current situation. The end-user gives the requirements to the development team then reduces the contact with them and responds late to requests. In the final stages of the project, Lebanese customers tend to inform the development of changes which causes frustration and stress among developers and lateness in delivery date. In addition, the political and economic situation in Lebanon carries high risks ranging from employee transportation, internet connectivity, and employee motivation leading to possible delays in delivery of software.

\subsection{Limitations}

A limitation of this research study is that the research sites were dictated by the availability of research sites and willingness to participate in this study; similarly for research participants. Thus, the study was conducted in a relatively small number of research sites. Yet the constant comparison technique used allows the accommodation of more data from new contexts; especially through the replication of the study in other developing countries. Further, using the TIC model as a basis for our analysis aided in the 
analysis of agile implementation in relation to the three elements. Difficulties were encountered when identifying an exciter from institutions to ICT. To achieve rigor and generalizability in qualitative research, four critical factors have been identified [23]: construct, internal and external validity, and reliability. They were achieved through collecting data using multiple sources of evidence, specifying unit of analysis, replication of the study, and use of a universal interview guide for participants, respectively.

\section{Conclusion}

Agile software development can enhance product quality and improve team productivity. In our study, we explore the practitioners' perceptions of the factors that enable or impede agile methods in developing countries through observing three companies in the Lebanese software sector.

Our study uses a qualitative research approach. The case study replicates the investigation in three research sites resulting in 31 semi-structured open-ended interviews with agile software development practitioners. The research employs the TIC model which interlinks institutional theory, the capabilities approach, and ICT to explore ICT4D. This model aids in understanding of agile software development process in developing countries through highlighting impediments and stimulators of the process.

The analysis reveals positive reinforcements which include detailed and inclusive sprint planning, a wide range of communication tactics used by stakeholders, and different communication technologies used to facilitate communication across geographical locations. In addition, the analysis of the model revealed impediments that demonstrate how, from an institutional perspective, there was low investment in ICT. A lack of agile process knowledge created implementation process gaps across the roles, artefacts and ceremonies of agile. In addition, we discovered from analyzing the impediments between ICT and capabilities the need to tackle the technical and social bottlenecks that include lack of customer involvement and risk mitigation of the critical political and economic situation. Future work will investigate adaptation of agile ceremonies in the Lebanese context and observe the challenges faced and benefits achieved.

\section{References}

1. Adolph S, Hall W, Kruchten P (2011) Using grounded theory to study the experience of software development. Empir Software Eng 16(4):487-513. doi:10.1007/s10664-010-9152-6.

2. Ahmed A, Ahmad S, Ehsan N et al (2010) Agile software development: Impact on productivity and quality. In: Anonymous 2010 IEEE International Conference on Management of Innovation \& Technology IEEE, p 287-291.

3. Akinnuwesi BA, Uzoka F, Olabiyisi SO et al (2013) An Empirical Analysis of End-User Participation in Software Development Projects in a Developing Country Context. The Electronic Journal of Information Systems in Developing Countries 58(1):1-25.

4. Bass J (2018) Tailoring in Large Scale Agile, Interview guide . https://salford.figshare.com/articles/Tailoring_in_Large-Scale_Agile_Interview_Guide/7122503.

5. Bass JM (2016) Artefacts and agile method tailoring in large-scale offshore software development programmes. Information and Software Technology 75:1-16.

6. Bass JM, Haxby A (2019) Tailoring Product Ownership in Large-Scale Agile Projects: Managing Scale, Distance, and Governance. IEEE Software 36(2):58-63. 
7. Bass JM, Nicholson B, Subhramanian E (2013) A framework using institutional analysis and the capability approach in ICT4D. Information Technologies \& International Development 9(1):pp. 19-35.

8. Bass J, Rahy S (2020) Agile Software Development in Lebanon. https://salford.figshare.com/articles/Agile_Software_Development_in_Lebanon/11887824.

9. Beck K, Beedle M, Van Bennekum A et al (2001) Manifesto for agile software development.

10. Blake E, Tucker W (2006) Socially aware software engineering for the developing world.

11. Dingsøyr T, Falessi D, Power K (2019) Agile Development at Scale: The Next Frontier. IEEE Software 36(2):30-38.

12. Dyba T, Dingsoyr T (2009) What Do We Know about Agile Software Development?. Software, IEEE 26(5). doi:10.1109/MS.2009.145.

13. Feghali T, Ahmed ZU, Halawani S (2007) Determinants of Lebanon's ICT export competitiveness: Evaluating a country's readiness to export ICT. Journal of Transnational Management 12(3):3-23.

14. Hoda R, Noble J, Marshall S (2013) Self-Organizing Roles on Agile Software Development Teams. Software Engineering, IEEE Transactions on 39(3):422-444. doi:10.1109/TSE.2012.30

15. IDAL (2018) Technology Sector in Lebanon Factbook 2018.

16. Mohallel AA, Bass JM (2019) Agile software development practices in Egypt SMEs: a grounded theory investigation. In: Anonymous International Conference on Social Implications of Computers in Developing Countries Springer, p 355-365.

17. Rahy S, Bass J (2019) Information Flows at Inter-Team Boundaries in Agile Information Systems Development. In: Anonymous European, Mediterranean, and Middle Eastern Conference on Information Systems, Limassol, Cyprus, 4-5 October.

18. Regassa Z, Bass JM, Midekso D (2017) Agile methods in Ethiopia: an empirical study. In: Anonymous International Conference on Social Implications of Computers in Developing Countries Springer, p 367-378.

19. Runeson P, Host M, Rainer A et al (2012) Case study research in software engineering: Guidelines and examples. John Wiley \& Sons.

20. Sommerville I (2016) Software Engineering, Global Edition. Pearson Education Limited, Harlow.

21. Tarhini A, Yunis M, El-Kassar A (2018) Innovative sustainable methodology for managing in-house software development in SMEs. Benchmarking: An International Journal 25(3):1085-1103.

22. UNDP (2016) Mind the Gap a Labour Needs Assessment for Lebanon.

23. Vaivio J (2012) Interviews - Learning the Craft of Qualitative Research Interviewing. European Accounting Review 21(1):186-189.

24. WIPO (2015) Study on the Economic Contribution of the Software Industry in Lebnaon. 